\title{
Grinding is effective in early orthodontic treatment of unilateral posterior crossbite
}

\author{
Is early treatment of unilateral posterior crossbite effective?
}

\author{
Petrén S, Bondemark L, Söderfeldt B. A systematic review \\ concerning early orthodontic treatment of unilateral posterior \\ crossbite. Angle Orthod 2003; 73:588-596
}

Data sources PubMed and the Cochrane Controlled Clinical Trials Register were searched. Reference lists from retrieved articles were also searched by hand for additional studies.

Study selection For inclusion, an article had to satisfy the following criteria: cases in primary and early mixed dentition with unilateral posterior crossbite; be a randomised controlled trials (RCT) or prospective and retrospective studies with concurrent untreated as well as normal controls; clinical trials must have compared at least two treatment strategies without any untreated or normal control group involved; and be written in English, German, French or a Scandinavian language.

Data extraction and synthesis Data extraction was carried out independently and in duplicate. Quality of the studies was assessed and categorised as low, medium and high. Interexaminer conflicts were resolved by discussion to reach a consensus.

Results The search identified 1001 articles, of which 12 met the inclusion criteria. Only two RCT of early treatment of crossbite have been performed, and these two studies support grinding as treatment in the primary dentition. As treatment strategies, quad-helix $(\mathrm{QH})$, expansion plates and rapid maxillary expansion (RME) are effective in the early mixed dentition with high success rates. There is, however, no scientific evidence available that shows which of the treatment modalities, that is, grinding, QH, expansion plates or RME, is the most effective.

Conclusions Most of the studies have the serious drawback of lack of power because of small sample size, bias and confounding variables, lack of method error analysis and blinding in measurements, and deficient or lack of statistical methods. Thus, the studies were not of high enough quality level to draw any evidence-based conclusions. Better-controlled RCT with sufficient sample sizes are needed to obtain reliable scientific evidence identifying which treatment is the most effective for early correction of unilateral posterior crossbite. Future studies should also include assessments of long-term stability as well as analysis of costs and side-effects of the interventions.

\section{Commentary}

The aetiology, consequences and treatment of posterior crossbite have been extensively studied but with some contradictory results. The present systematic review aims to assess the orthodontic treatment effects on unilateral crossbites. The quality of a systematic review is dependent upon the quality of the studies from which the conclusions were drawn. Several methodological deficiencies were identified in almost all the published studies, which will limit the scientific soundness of their conclusions.

Regarding the search strategy in the present systematic review, a relatively common limitation was found. The use of only one database (PubMed) in the search for the available literature potentially allows key articles to be missed. Several other databases, at least also Medline and Embase, should be included so that readers can see every effort

Address for correspondence: Lars Bondemark, Department of Orthodontics, Faculty of Odontology, Malmö University, S-205 06 Malmö, Sweden. E-mail: lars.bondemark@ od.mah.se was made to find the available evidence. ${ }^{1,2}$ In fact, two systematic reviews ${ }^{3,4}$ published describing the long-term skeletal and dental effects of RME in crossbite cases showed that using only PubMed would have resulted in fewer than $50 \%$ of potential articles. When slow maxillary expansion ${ }^{5}$ was used almost $75 \%$ were selected by PubMed.

The authors searched for articles written in English, German, French and Scandinavian languages. This is a comprehensive search strategy which would normally cover almost all possible hits, but in the case of crossbite treatment there are significant numbers of articles published in Portuguese, some of which are of sound methodological quality. Again, the above-mentioned systematic reviews of RME-effects found that around $20 \%$ of the potentially useful articles were obtained via the Lilacs database (Latin-American journals database). Lilacs is predominantly Spanish and Portuguese which can be a disadvantage. But any language limitation can result in bias in metaanalysis. ${ }^{6}$

Regarding the final article selection, it is not completely clear how the authors went from the abstracts to the final selection of articles. Although unilateral crossbite treatment was sought, it is not obvious when and how unilateral crossbite was used as a selection criterion. One important point that was not properly considered was that a significant number of unilateral crossbites are bilateral once the individual is in centric relationship. If true unilateral crossbites were separated from pseudo-unilateral crossbites, the results and conclusions of the present systematic review would have been even more limited.

\section{Practice points}

- The available evidence is of low quality.

- From the available evidence, grinding of interferences appears to be the initial treatment of choice for unilateral posterior crossbites. - Alternative approaches (quad-helix, removable and fixed expansion plates) offered promising results but there are not yet data relating to their long-term stability.

\section{Carlos Flores-Mir}

Faculty of Medicine and Dentistry, Dentistry/Pharmacy Center, University of Alberta, Edmonton, Alberta, Canada

1. Moher D, Cook DJ, Eastwood S, Olkin I, Rennie D, Stroup DF. Improving the quality of reports of meta-analyses of randomised controlled trials: the QUOROM statement. QUOROM Group. Br J Surg 2000; 87:1448-1454.

2. Egger M, Smith GD, Schneider M. Systematic reviews of observational studies. In Systematic Reviews in Health Care: Meta-analysis in Context Edited by Egger M, Smith GD, Altman DG London: BMJ; 2003; 211-227.

3. Lagravere M, Major P, Flores-Mir C. Long-term dental arch changes after rapid maxillary expansion treatment: a systematic review. Angle Orthod 2005; 75:151-157.

4. Lagravere $M$, Major $P$, Flores-Mir $C$. Long-term skeletal changes with rapid maxillary expansion: a systematic review. Angle Orthod 2005; 75:833-839.

5. Lagravere M, Major P, Flores-Mir C. Skeletal and dental changes with slow maxillary expansion: a systematic review. J Am Dent Assoc 2005 (in press).

6. Juni P, Holenstein F, Sterne J, Bartlett C, Egger M. Direction and impact of language bias in meta-analyses of controlled trials: empirical study. Int J Epidemiol 2002; 31:115-123.

Evidence-Based Dentistry (2005) 6, 24.

doi:10.1038/sj.ebd.6400315 